\title{
Model predictions for bone conduction perception in the human
}

\author{
Stefan Stenfelt \\ Linköping University Post Print
}

Tweet

N.B.: When citing this work, cite the original article.

Original Publication:

Stefan Stenfelt Model predictions for bone conduction perception in the human, 2016, Hearing Research, 15, 30076-30079.

http://dx.doi.org/10.1016/j.heares.2015.10.014

Copyright: Elsevier

http://www.elsevier.com/

Postprint available at: Linköping University Electronic Press

http://urn.kb.se/resolve?urn=urn:nbn:se:liu:diva-125727

(9) (1) $\Theta \Theta$ 
Model predictions for bone conduction perception in the human

Stefan Stenfelt

Department of Clinical and Experimental Medicine

Linköping University

58185 Linköping, Sweden

Email: Stefan.stenfelt@liu.se

Phone: +4613284798 


\section{Abstract}

Five different pathways are often suggested as important for bone conducted (BC) sound: (1) sound pressure in the ear canal, (2) inertia of the middle ear ossicles, (3) inertia of the inner ear fluid, (4) compression of the inner ear space, and (5) pressure transmission from the skull interior. The relative importance of these pathways was investigated with an acousticimpedance model of the inner ear. The model incorporated data of BC generated ear canal sound pressure, middle ear ossicle motion, cochlear promontory vibration, and intracranial sound pressure. With BC stimulation at the mastoid, the inner ear inertia dominated the excitation of the cochlea but inner ear compression and middle ear inertia were within $10 \mathrm{~dB}$ for almost the entire frequency range of 0.1 to $10 \mathrm{kHz}$. Ear canal sound pressure gave little contribution at the low and high frequencies, but was around $15 \mathrm{~dB}$ below the total contribution at the mid frequencies. Intracranial sound pressure gave responses similar to the others at low frequencies, but decreased with frequency to a level of $55 \mathrm{~dB}$ below the total contribution at $10 \mathrm{kHz}$. When the BC inner ear model was evaluated against AC stimulation at threshold levels, the results were close up to approximately $4 \mathrm{kHz}$ but deviated significantly at higher frequencies. 


\section{Highlights}

- A model for BC simulates the contribution from 5 components.

- The inner ear compression and inertia and middle ear inertia are most important.

- The contribution from intracranial sound pressure is insignificant at higher frequencies.

- The model provides similar BM excitation for AC and BC threshold stimulation up to $4 \mathrm{kHz}$.

\section{Keywords}

Bone conduction, inner ear model, fluid inertia, inner ear compression, middle ear inertia

\section{Abbreviations}

AC - Air conduction

$\mathrm{BC}$ - Bone conduction

BM - basilar membrane

CA - Cochlear aqueduct

CSF - Cerebrospinal fluid

OW - Oval window

RW - Round window

SV - Scala vestibule

ST - Scala tympani

VA - Vestibular aqueduct 


\section{Introduction}

Today, it is well accepted that bone conducted (BC) sound excites the inner ear and creates a traveling wave on the basilar membrane (BM) similar to air conduction (AC) stimulation. This notion is based on the ability to cancel a BC tone by an AC tone (Stenfelt, 2007; von Békésy, 1932), the ability to generate distortion-product otoacoustic emissions using BC stimulation (Purcell et al., 1998; Watanabe et al., 2008), and the similarity of BM vibration pattern with AC and BC excitation (Stenfelt et al., 2003a). However, the way the sound is transmitted from the excitation position, often at the mastoid or forehead of the skull, to the inner ear and causing the BM vibration is not clarified.

Through history, several explanations have been presented for the perception of BC sound. Early investigators proposed one or two contributors for the perception of BC sound (Bárány, 1938; Herzog, 1926; Krainz, 1926) while von Békésy suggested contribution from the outer, middle and inner ear (von Békésy, 1960). The number of contributors and their relative importance has varied over time and in total seven contributors for BC sound perception was proposed by Tonndorf (1966) from studies in cats. Lately, five different contributors for BC sound have been suggested (Stenfelt, 2011; Stenfelt et al., 2005b). These five contributors are (1) generation of sound pressure in the ear canal, (2) relative motion of the middle ear ossicles caused by inertial effects from the mass of the ossicles, (3) inertial forces acting on the inner ear fluid, (4) compression and expansion of the cochlear space, and (5) transmission of sound pressure from the skull interior.

Several investigations have been presented to experimentally estimate the contribution and its relative importance from these five contributors. The one component that is easiest to measure is the contribution from sound pressure in the ear canal. This contribution can be estimated by measuring the ear canal sound pressure during $\mathrm{AC}$ and $\mathrm{BC}$ stimulation and compare its level at hearing thresholds. Huizing (1960) found that the sound pressure in the 
ear canal was greater with AC stimulation than with BC stimulation causing the same hearing sensation, at least for frequencies above $500 \mathrm{~Hz}$. Khanna et al. (1976) estimated the contribution from the ear canal sound pressure by cancelling an AC tone by a BC tone. They reported the AC and $\mathrm{BC}$ sounds to be similar for frequencies up to $1.5 \mathrm{kHz}$, while above 1.5 $\mathrm{kHz}$ the AC tone was 10 to $20 \mathrm{~dB}$ greater than the $\mathrm{BC}$ ear canal sound at cancellation. The caveat with their study is that they occluded the ear to provide the AC sound, and thereby increased the low-frequency ear-canal sound pressure generated by BC, known as the occlusion effect (Huizing, 1960; Reinfeldt et al., 2013; Stenfelt et al., 2007). Another approach was used in Stenfelt et al. (2003b) where the umbo (tip of the malleus) vibration was compared to AC and BC generated ear canal sound pressure. It was concluded in that study that $\mathrm{AC}$ sound in the ear canal gave approximately $10 \mathrm{~dB}$ lower umbo velocity compared to BC generated sound in an open ear canal. This indicates that there are other mechanisms driving the relative motion of the middle ear ossicles than the sound in the ear canal during BC stimulation, and that the sound pressure in the ear canal generated by BC is some $10 \mathrm{~dB}$ below the contribution from the middle ear, most probably driven by inertia of the middle ear ossicles. However, when the ear canal was occluded, the sound pressure driven umbo motion in Stenfelt et al. (2003b) was equal for AC and BC sound at frequencies below $1.2 \mathrm{kHz}$ indicating that for low frequencies, the ear canal sound pressure is a dominant contributor for hearing BC sound when the ear is occluded.

The relative importance of the other four contributors are more difficult to assess. One attempt to estimate the contribution from the middle ear inertia has been done by comparing the motion of the ossicles at hearing thresholds when stimulation was by AC and BC (Röösli et al., 2012; Stenfelt, 2006). Both studies indicate greater ossicle motion with AC stimulation than $\mathrm{BC}$ stimulation for frequencies at $3 \mathrm{kHz}$ and below, but the opposite at higher frequencies. The interpretation was that the middle ear inertia was not dominant at 
frequencies below $3 \mathrm{kHz}$ for BC stimulation. This is somewhat opposite to interpretations of clinical findings of otosclerosis of the stapes footplate, where a depression of the BC thresholds at and around $2 \mathrm{kHz}$ of up to $20 \mathrm{~dB}$ is seen, often termed the Carhart notch (Carhart, 1971). This depressed BC thresholds has been attributed to the lack of middle ear inertia (Tonndorf, 1966) as the ossicles resonance frequency for BC stimulation is close to 2 kHz (Homma et al., 2009; Stenfelt et al., 2002). However, in a model simulation of BC excitation of the inner ear (Stenfelt, 2015), the depressed BC thresholds close to $2 \mathrm{kHz}$ could be simulated as a result of increased impedance at the oval window (OW). In those simulations, the effect of middle ear inertia was not included but the Carhart notch was seen as a result of increasing the impedance at the OW seen from inside the inner ear.

The latter is a general problem for experimental manipulations investigating BC sound. It is nearly impossible to isolate one contributor without affecting one or more of the other contributors and the results become difficult to interpret. One way to circumvent this problem is to use a model that can simulate BC excitation. Several such models have been devised to investigate a specific aspect of BC stimulation, for example the occlusion effect (Brummund et al., 2014; Schroeter et al., 1986; Stenfelt et al., 2007), middle ear inertia (Homma et al., 2009; Williams et al., 1990), and inner ear fluid inertia and compression (Bohnke et al., 2006; Kim et al., 2011; Schick, 1991; Stenfelt, 2015). These models are often specific meaning they only investigate a single or a couple of phenomena and not the complete response of the ear to BC stimulation. Another important aspect is the excitation of the BC sound itself. The vibration of the bone surrounding the ear is complex showing several modes of wave transmission with translational as well as rotational motion in all three dimension (EegOlofsson et al., 2013; McKnight et al., 2013; Stenfelt et al., 2005a; Stenfelt et al., 2000). Most models have used a simple excitation pattern preventing extrapolations of the results to clinical reality. One exception is a three-dimensional whole-head finite element model for BC 
sound (Taschke et al., 2006). However, that model have not been used to investigate contributors for BC sound in detail.

Recently, an inner ear model for BC sound was presented that used excitation based on the motion of the inner ear boundary (Stenfelt, 2015). The motion of the surrounding bone was based on experimental investigations of bone vibration transmission in the skull base, and the model was able to simulate several experimental and clinical findings for BC sound reported in the literature. That model is the core for the current simulations and is extended to be able to predict relative importance from the five components listed above.

The aim of the current study is to use a model to predict the relative importance of five contributors for BC sound in the human. The model is based on mechano-acoustic impedances of the middle and inner ear as well as data from experimental studies of BC sound in the human reported in the literature. 


\section{The model}

\subsection{Ear model}

The model depicted in Fig 1 shows a simplified sectional image of the ear. This layout is used for the predictions of the five components for BC hearing listed above. Figure 1 does not constitute a model in itself, but shows the steps and pathways used to compute predictions of the five components. The layout comprises the ear canal, the middle ear, the inner ear, the skull bone surrounding the peripheral auditory organ, and the skull interior composed of the brain surrounded by the cerebrospinal fluid (CSF). Also indicated in Fig. 1 is the compliant pathways between the inner ear and the skull interior, the cochlear aqueduct (CA) and the vestibular aqueduct (VA).

All computations is made for a mastoid placement of the BC transducer, approximately at the audiological placement of a BC transducer, 20-30 mm behind the ear canal opening. The data used are for a transducer attached to the skull bone, but similar results are expected for positions on the skin covered bone at or close to the used position. Other stimulation positions, for example stimulation at the forehead or at positions dominated by pure soft tissues, would alter the relative importance of the pathways making the current predictions invalid.

Three computational pathways are indicated by arrows in Fig. 1. Pathway 1 (blue arrows) is for the estimation of the ear canal sound pressure component, where pathway $1 \mathrm{~A}$ indicates the ear canal sound pressure during BC excitation, and pathway 1B shows the transmission of the ear canal sound pressure to the inner ear via the middle ear similar to that with AC sound. Pathway 2 (purple arrow) indicates sound transmission from the stimulation position to the vibration of the bone surrounding the inner ear. This pathway is used to compute the predictions for middle ear inertia and the two inner ear components, fluid inertia and inner ear 
compression. The wave motion used for these computations is a longitudinal wave with constant wave speed. Even if several studies have indicated the wave motion in the cranial vault to be complex with plate waves or spherical waves (McKnight et al., 2013; Tonndorf et al., 1981), wave motion in the skull base seem to be dominated by longitudinal waves with a wave speed of approximately 400 m/s (Eeg-Olofsson et al., 2008; Stenfelt et al., 2005a). Therefore, the wave motion for pathway 2 is a longitudinal wave with wave speed $400 \mathrm{~m} / \mathrm{s}$. The third pathway (red arrow) is the pathway for stimulation through the skull interior. This pathway is often experimentally investigated by a direct excitation of the soft tissues/brain (Sohmer et al., 2000), but is here modeled as a transmission via the skull bone (pathway 3A) and then a transmission via the VA to the inner ear (pathway 3B). The rationale for this is explained in detail below.

\subsection{Inner ear model}

Figure 2 shows the impedance model of the inner ear used for the predictions. This model is essentially the same model used in a previous study (Stenfelt, 2015). In that study only predictions of the inner ear was computed (fluid inertia and inner ear compression). To enable predictions from all five components, the model is slightly changed. Moreover, some of the parameters are updated compared to the parameters in the Stenfelt (2015) study.

The impedances, pressure generators, and fluid flow (volume velocity) generators are overlaid a tapered 2-dimensional geometry of the inner ear consisting of three compartments, vestibule, scala vestibuli (SV) and scala tympani (ST), and four compliant inlets and outlets of the inner ear, the round window (RW), OW, VA, and CA. It should be noted that the relative dimensions of the $2 \mathrm{D}$ inner ear model is not drawn correctly, but is adjusted to show the principle of the connection between the geometry and the impedances and generators. For the different contributors, different generators are active. The fluid generator $U_{S F}$ is caused 
by the motion of the stapes footplate, is used for predictions of the two components ear canal sound pressure and middle ear inertia, and is short circuited for the other three components. The three pressure generators $P_{V}, P_{S V}$, and $P_{S T}$ are active for computations of the fluid inertia and short-circuited for all other components. The fluid generators $U_{V}, U_{S V}$ and $U_{S T}$ are active for computing the inner ear compression response and open-circuited otherwise, while the pressure generator $P_{I C}$ is used to compute the response from the intracranial pressure and is short-circuited for all other components.

The impedances in Fig. 2 are based on the mass of the fluid inside the inner ear, estimates from the literature, and fluid filled ducts. The fluid generators $U_{V}, U_{S V}$ and $U_{S T}$ are modeled as injecting the fluid in the center of each compartment, and the fluid mass of SV, ST and the vestibule is divided equally at the two sides of each fluid generator. The impedances, parameter values, and their references are presented in Table 1 . The masses $M_{S T}, M_{S V}$, and $M_{V}$ as well as the impedances of the ducts for CA and VA (Table 1) are based on the density of the inner ear fluid (modeled as water) and the associated dimensions. Details of these computations can be found in Stenfelt (2015).

\subsection{Contribution from ear canal sound pressure}

According to Fig. 1, the contribution from the ear canal sound pressure is estimated in two steps. First, the ear canal sound pressure for BC excitation is derived (arrow 1A, Fig. 1), and second, the transmission of this sound in the ear canal to a motion of the fluid in the inner ear is computed (arrow 1B, Fig. 1). The ear canal sound pressure during BC excitation is obtained from Fig. 3 in Stenfelt et al. (2003b). The data used is for the open ear canal. In that study, the sound pressure in the ear canal was obtained just in front of the eardrum while the stimulation was at the mastoid bone, some $35 \mathrm{~mm}$ behind the ear canal opening. That sound pressure is then transmitted to the inner ear. To calculate that transmission, the stapes 
footplate displacement in Fig. 3 in Hato et al. (2003) is used. That data shows the footplate displacement for a sound pressure at the eardrum. The displacement of the footplate is converted to volume displacement at the OW by multiplying the footplate displacement with the area of the footplate. The area used is $3.85 \mathrm{~mm}^{2}$ which was the average area of 15 footplates in Stenfelt et al. (2004).

To convert the volume displacement at the OW to an excitation of the BM, the impedance network in Fig. 2 is used. In Fig. 2, the OW volume displacement is converted to volume velocity and the volume velocity source $U_{S F}$ excites the model. Using the model, the volume displacement of the fluid over the BM is computed. This volume displacement is derived as the volume velocity through the impedance $Z_{C}$ and then integrated to obtain the volume displacement (or divide by $j \omega$ in the frequency domain). This volume displacement is here

used as the drive for the hearing of BC sound. The volume displacement of the inner ear fluid over the BM caused by the sound in the ear canal during BC stimulation of $1 \mathrm{~N}$ at the mastoid is shown in Fig. 3.

\subsection{Contribution from the middle ear}

The contribution from the middle ear is based on the inertial effects of the ossicle mass. This inertia means that at low frequencies, the ligaments and eardrum dominates and the motion of the ossicles are similar to the motion of the bone surrounding the middle ear. However, at higher frequencies the inertia dominates and the ossicles lag the motion of the surrounding bone causing a relative motion between the ossicles and the bone. The relative motion between the stapes footplate and the adjacent bone was measured in Stenfelt et al. (2002) and mean data from Fig. 3b in that study is used as the relative motion between the stapes and promontory bone here. That relative motion is used for the source $U_{S F}$ in Fig. 2 by multiplying the motion with the stapes footplate area of $3.85 \mathrm{~mm}^{2}$ as described above. 
To obtain the contribution from the middle ear inertia the relative motion is multiplied by the motion of the bone surrounding the middle ear. No specific measure of the bone surrounding the middle ear was found in the literature, so the motion of the cochlear promontory was used. The cochlear promontory is close to the OW which is the position of the stapes footplate. Several measurements of the promontory motion has been obtained and for the simulations here the average from two datasets is used, the cadaver head measurements of Stenfelt et al. (2005a) and Eeg-Olofsson et al. (2008). From the Stenfelt et al. (2005a) study the average data from Fig. 6 with Occiput I-3 position are used and from the Eeg-Olofsson et al. (2008) study the average data from Fig. 3 are used. The promontory displacement with 1 $\mathrm{N}$ excitation is shown in Fig. 4.

The contribution from the middle ear can be estimated from the inner ear model in Fig. 2 where the motion of the stapes footplate is converted to volume velocity and used for the $U_{S F}$ source. The volume displacement at the BM is then computed similar to the way it was done for the ear canal sound pressure contribution, and the result is displayed in Fig. 3.

\subsection{Contribution from the skull interior}

The theory for this contributor is that the vibrations, either in the bone or in the soft tissues, are transmitted to the interior of the skull, i.e. the brain and CSF. It is here modeled as sound transmission from the stimulation position to the inside of the head (Arrow 3A, Fig. 1).

Recently, intracranial sound pressure measurements was presented by Roosli et al. (2015). In that study, the intracranial sound pressure during BC stimulation was measured at the center of four skulls with a hydrophone. The average of the intracranial sound pressure from these four heads when stimulated at the mastoid are used as the pressure source $P_{I C}$ in Fig. 2. The data in Roosli et al. (2015) are only presented for frequencies above $300 \mathrm{~Hz}$ so the computation of this contributor is only done for frequencies between 0.3 and $10 \mathrm{kHz}$. 
The intracranial sound pressure affects both aqueducts, the VA and CA. Since the measurements of the intracranial sound pressure were done at a distance (approximately 50 $\mathrm{mm}$ ) from the aqueducts it is not known if there were any phase differences between the pressures at the two openings. However, when computing the contribution from the intracranial sound pressure, the effect from the sound pressure at the CA was insignificant for the result, irrespectively if it was in-phase, out-of phase, or zero compared to the pressure at the VA. Therefore, for the present computations, the intracranial sound pressure was a source for the VA solely, as indicated by the pressure source $P_{I C}$ in Fig. 2.

The contribution from the intracranial sound pressure was estimated by computing the fluid flow at the BM using the model in Fig. 2 and then integrate the volume flow to volume displacement. The volume displacement at the BM from intracranial pressure are presented in Fig. 3 together with the estimates for the other contributors.

\subsection{Contribution from the inner ear}

The fluid inertia and inner ear compression is based on the motion of the inner ear boundary. The computations for these two contributors were presented in detail in Stenfelt (2015) and only an overview of the computations are presented here. The updated computations are explained in detail.

Essential for the computations is that the wave motion in the bone is assumed to be a longitudinal wave with a wave speed of $400 \mathrm{~m} / \mathrm{s}$. With this type of wave, the motion of the inner ear space can be divided into two types, (1) common motion and (2) differential motion (see Fig. 2 in Stenfelt (2015)). In common motion, the inner ear boundaries move with the same amplitude and phase causing the same motion for the entire inner ear. This motion gives rise to the inertial forces of the inner ear fluid. The pressure sources $P_{V}, P_{S V}$, and $P_{S T}$ can be 
computed from the mass $m$ of the fluid, the cross-sectional area $A$, and the displacement $D$ of the inner ear caused by the stimulation as

$P=\frac{-\omega^{2} \cdot D \cdot m}{A}$

The geometries for computing the masses and the and pressure sources are shown in Fig. 2 and are taken from Purcell et al. (2003) and Seibel et al. (2006). The middle ear impedance, $Z_{M E}$, is the impedance seen from inside the inner ear at the OW. This impedance was estimated by Puria (2003) and is used here. However, in the estimate done by Puria (2003) the loading of the VA on the measurement was not considered, and the $Z_{M E}$ used for the present computations is adjusted for the load of the VA. The impedance of the VA $\left(Z_{V A}\right)$ is modeled as two ducts in series, one $1.5 \mathrm{~mm}$ long with a $0.25 \mathrm{~mm}$ diameter and one $8.5 \mathrm{~mm}$ long with $0.5 \mathrm{~mm}$ diameter. These are slightly smaller diameters than in the Stenfelt (2015) study, but still according to the geometries presented for the VA in Clemis et al. (1968). The impedance for the CA $\left(Z_{C A}\right)$ equals that presented in Stenfelt (2015) and is modelled as a $10 \mathrm{~mm}$ tube of $0.15 \mathrm{~mm}$ diameter, according to geometries presented by Gopen et al. (1997). $Z_{B M}$, that is part of $Z_{C}$ in Fig. 2, was estimated by Puria (2003) and $Z_{R W}$ was estimated by Merchant et al. (1996). Using these impedances and pressure generators, the volume displacement through the BM for inner ear fluid inertia can be computed and is shown in Fig. 3.

The last contributor, compression and expansion of the inner ear space, is caused by the space change of the inner ear during the $\mathrm{BC}$ wave. The fluid of the inner ear is considered as incompressible, and the space alteration forces the fluid towards the compliant inlets and outlets of the inner ear during the compression phase. The expansion phase causes an opposite fluid flow. Each compartment in the inner ear has an associated fluid volume velocity generator $(U)$. The fluid volume velocity from each generator is computed as the 
space alteration of the specific compartment due to the differential motion mentioned above. A more detailed explanation for the fluid generation can be found in Stenfelt (2015).

The layout in Fig. 2 shows the SV and ST to be tapered. According to Braun et al. (2012), SV height is $1.2 \mathrm{~mm}$ at the base that become $0.6 \mathrm{~mm}$ at the base, and the width is $1.78 \mathrm{~mm}$ at the base that become $1.58 \mathrm{~mm}$ at the apex. This result in an effective fluid generation that is 0.70 of a straight box with the dimensions at the base. The tapering effect of the ST is 0.58 where the height and width at the base are $1.39 \mathrm{~mm}$ and $2.47 \mathrm{~mm}$ that become $0.57 \mathrm{~mm}$ and 1.62 $\mathrm{mm}$ at the apex. Another effect that diminish the effective compression is that in reality, the cochlea is coiled while shown as uncoiled in Fig. 2. For simplicity, the cochlea is considered to be circular in the computations, which can be seen as a first order of approximation from the straight box to a coiled version of the cochlea. Without including any phase differences between the different parts of the circular cochlea, the compression for a one-dimensional BC sound wave is 0.705 for a circular dimension compared to the straight box. This is included in the computations of the fluid generation in the volume velocity sources for SV and ST.

According to the above, the fluid displacement at the BM from inner ear compression is shown in Fig. 3. 


\section{Model simulations}

The contribution from the five components considered most important for BC hearing in the human when the BC stimulation is a $1 \mathrm{~N}$ excitation at the mastoid bone is shown in Fig. 3. According to that figure, the inner ear components and the middle ear inertia seem to be important for the frequency range investigated while intracranial pressure is important at frequencies below $0.7 \mathrm{kHz}$ and ear canal sound pressure only seems important at mid frequencies, between $0.7 \mathrm{kHz}$ and $3 \mathrm{kHz}$.

The results in Fig. 3 are plotted according to their relative importance. The total contribution from all components is computed as the square-root out of the sum of all components squared. This neglects the phase of each contributor and adds the total energy from the 5 components. Then, each component is related to the total contribution and shown in Fig. 5. This figure shows that for the lowest frequencies, at $0.4 \mathrm{kHz}$ and below, inner ear compression dominates the response while at higher frequencies, fluid inertia causes the greatest excitation of the BM. The middle ear inertia component is some 5 to $10 \mathrm{~dB}$ below the total contribution at frequencies above $0.5 \mathrm{kHz}$ and $20 \mathrm{~dB}$ below at $0.1 \mathrm{kHz}$. The intracranial sound pressure contribution is $7 \mathrm{~dB}$ below the total contribution at $0.3 \mathrm{kHz}$, which is the lowest frequency measured for this contributor. It falls nearly monotonically with frequency and ends at $55 \mathrm{~dB}$ below the total contribution at $10 \mathrm{kHz}$. The ear canal component rises with frequency from $30 \mathrm{~dB}$ below the total contribution at $0.1 \mathrm{kHz}$ to $12 \mathrm{~dB}$ below the total contribution at $0.6 \mathrm{kHz}$. It then falls slowly with frequency to $20 \mathrm{~dB}$ below the total contribution at $3 \mathrm{kHz}$ where it drops rapidly and stays at around $35 \mathrm{~dB}$ below the total contribution at the highest frequencies.

The model predicts the excitation of the BM during BC stimulation at the mastoid. This means that the model can be compared to AC stimulation of the BM as well. If the model predictions are correct, the BM excitation of BC stimulation at BC thresholds should equal 
the BM excitation of AC stimulation at AC thresholds. This comparison was done according to the following. First, the hearing thresholds for BC stimulation was obtained from the standard (ISO:389-3, 1994). These data are for hearing thresholds with a bone transducer applied to the skin-covered mastoid, and they were transformed to force thresholds at the mastoid bone similar to what was done in Stenfelt (2006). According to that transformation, the BM displacement was computed as the total displacement for all contributors with threshold stimulation. Next, the BM displacement was estimated for AC stimulation at threshold levels. First, the ear canal minimum audible sound pressure estimate from Killion (1978) was used. This is an estimate of the ear canal sound pressure that is required to evoke a hearing perception. This ear canal sound pressure is converted to an inner ear pressure using the middle ear gain function of Aibara et al. (2001). In that study, the inner ear sound pressure was obtained by a hydrophone in the SV or vestibule. It was here interpret as a sound pressure between $Z_{V}$ and $Z_{S V}$ in Fig. 2. This sound pressure could be used to derive a volume displacement at the BM as caused by the AC stimulation at threshold. These two estimates, the $\mathrm{AC}$ driven $\mathrm{BM}$ displacement at hearing thresholds and the $\mathrm{BC}$ driven $\mathrm{BM}$ displacement at hearing thresholds according to the model are presented in Fig. 6. In this figure, the relative BM displacement at $\mathrm{AC}$ thresholds compared to at $\mathrm{BC}$ thresholds is depicted. This function shows that at the lowest frequencies, the model predicts around $20 \mathrm{~dB}$ greater BM displacement for BC stimulation than for AC stimulation. At $250 \mathrm{~Hz}$ the difference is $8 \mathrm{~dB}$ that diminish with frequency and become $0 \mathrm{~dB}$ at $800 \mathrm{~Hz}$. Then, the difference is close to $0 \mathrm{~dB}$ up to $2.5 \mathrm{kHz}$ where it increases and becomes close to $15 \mathrm{~dB}$ at the highest frequencies. 


\section{Discussion}

The relative importance of five contributors for BC hearing in the human was investigated by an ear model and measurements presented in the literature of bone vibration, ear canal sound pressure, middle ear ossicle motion, and intracranial sound pressure. The model itself is a simplification of the complex 3D anatomy and the simulations are based on the impedances of the different entities in the inner ear, estimated either from the geometry and physical properties, or from estimates of the inner ear impedances found in the literature.

Central to this model is that the bone vibration at the inner ear is dominated by longitudinal vibration. The inner ear model itself was investigated in Stenfelt (2015) and it was shown that this model can predict experimental findings of BC sound. However, most of those comparisons was done for relative differences, i.e. the difference between the normal and an experimental condition. Here, the model was evaluated with the motion of the BM for AC and BC threshold stimulation. The results in Fig. 6 shows that the motion of the BM at threshold for $\mathrm{AC}$ and $\mathrm{BC}$ stimulation was within $10 \mathrm{~dB}$ for frequencies between 0.25 and 4 $\mathrm{kHz}$, and differ up to $20 \mathrm{~dB}$ at the highest and lowest frequencies. The model predictions were based on several assumptions. One such assumption was that the bony motion was longitudinal with a constant wave speed of $400 \mathrm{~m} / \mathrm{s}$. There is no support in the literature that the wave speed of bony motion is constant throughout the entire frequency range. Several studies of the velocity of the bone vibration have indicated different wave speed at the low compared to high frequencies. Both Tonndorf et al. (1981) and Franke (1956) indicated lower wave speed at low frequencies. However, Brandt (1989) pointed out that the skull behaves nearly as a rigid body at low frequencies, since the velocity of propagation approaches infinity for low frequencies. This behavior was included in the comparison between AC and BC driven BM motion. The dashed line in Fig. 6 shows the simulation results with a higher wave speed, here $1000 \mathrm{~m} / \mathrm{s}$. In this case, the low-frequency difference between AC and BC 
stimulation is less, but there is no difference at high frequencies. Consequently, the bone vibration at low frequencies may have a greater velocity than that used for the current simulation. It should be noted that a lower wave speed, as suggested by Tonndorf et al. (1981) and Franke (1956), would result in greater difference between the low-frequency AC and $\mathrm{BC}$ driven BM motion as reported in Fig. 6. One way to further investigate the sound transmission in the skull base where the inner ear is situated and its impact on the inner ear boundary motion, is to use anatomically correct finite element models of the whole human head (Kim et al., 2014b; Taschke et al., 2006). To date, no such model have been presented with that ability.

Another parameter that influences the comparison of the $\mathrm{AC}$ and $\mathrm{BC}$ driven $\mathrm{BM}$ motion in Fig. 6 is the magnitude of the cochlear promontory motion. The simulations are based on the average of two datasets (Eeg-Olofsson et al., 2008; Stenfelt et al., 2005a). Both these datasets are obtained in cadaver heads. There may be differences between the vibration transmission in a cadaver head and a live human skull. In Fig. 4, the promontory motion measured in live humans is shown (Eeg-Olofsson et al., 2013). That data are obtained with the stimulation applied at the skin covered mastoid and were used for the comparison between AC and BC BM motion at threshold in Fig. 6 (dotted line). According to the result, live human data gives better correspondence (closer to $0 \mathrm{~dB}$ ) at high and low frequencies, but worse similarity at mid frequencies. Consequently, the result for the comparison depend on the choice of parameter values as well as experimental datasets.

The AC transmission was not investigated in detail here, but some of the discrepancy may be due to uncertainties in the AC transmission pathway. For example, the high frequency discrepancy correlate with the high-frequency deterioration of the middle ear transfer function found in most temporal bone experiments (Aibara et al., 2001; Hato et al., 2003). However, there are arguments that there is a difference for live humans with a broadband 
middle ear transfer function that is relatively independent of frequency at high frequencies (Huber et al., 2001; Ruggero et al., 2003).

Puria et al. (1997) measured the vestibular sound pressure in human temporal bones when the stimulation was a sound pressure in the ear canal. Their results indicated that the vestibular sound pressure was close to $28 \mathrm{~dB}$ SPL at hearing thresholds. When the data in Fig. 6. are converted to sound pressures, the BC differential sound pressure between SV and ST is between 27 and $32 \mathrm{~dB}$ SPL for frequencies above $450 \mathrm{~Hz}$ but greater at the lowest frequencies. The AC SV pressure is between 23 and $37 \mathrm{~dB}$ SPL for the entire frequency range. This indicates that the $\mathrm{BC}$ model is reasonable apart from the lowest frequencies where it seem to overestimate the excitation of the BM. Moreover, if the Puria et al. (1997) data are used instead of the Aibara et al. (2001) data in Fig. 6., the high frequency difference between the AC and BC BM excitation diminish and becomes close to $10 \mathrm{~dB}$. In conclusion, the discrepancy between the AC and BC results in Fig. 6. may originate in uncertainties of both transmission modalities.

\subsection{Relative importance of the five components}

Figures 3 and 5 shows that there are relatively small differences for the three contributors fluid inertia, middle ear inertia and inner ear compression. With a few exceptions, they are within $10 \mathrm{~dB}$ of each other. This difference is generally smaller than the difference between the fluid inertia and the inner ear compression reported in the previous model (Stenfelt, 2015). One reason for this can be the more realistic computation of the compression of the cochlear part including tapering and a circular geometry. Also, the slightly greater impedance of the VA in the current model compared to Stenfelt (2015) reduce the importance of the fluid inertia. Even so, for the greatest part of the frequency range presented here, fluid inertia dominates the response. 
The estimation of the contribution from the middle ear was done from measurements of the stapes motion during bone vibration in human temporal bones (Stenfelt et al., 2002). In those measurements, the inner ear was intact with fluid inside. Consequently, the measured stapes motion could be due to the inertial effects of the middle ear ossicles restrained by the ligaments, but it could also be influenced by the fluid inside the inner ear. Therefore, the contribution from the middle ear may be overestimated or underestimated depending on the action from the fluid on the OW. Homma et al. (2009) devised a finite-element model of the middle ear where the fluid inside the inner ear was modeled as a load on the stapes motion, and no action from the inner ear was present. When the stapes motion from that model was compared with the motion measured by Stenfelt et al. (2002), there was 10 to $15 \mathrm{~dB}$ less relative motion at frequencies below $1 \mathrm{kHz}$ in the model compared to the measurement, 5 to $10 \mathrm{~dB}$ greater motion for the model at frequencies between 1 and $2 \mathrm{kHz}$, and around $5 \mathrm{~dB}$ less motion for the model compared to the measurements at frequencies above $2 \mathrm{kHz}$. This means that if the finite-element model motion is correct, the current model overestimates the middle ear contribution at low and high frequencies, and underestimates the contribution at frequencies between 1 and $2 \mathrm{kHz}$. If 5 to $10 \mathrm{~dB}$ motion is added to the middle ear ossicle contribution for frequencies between 1 and $2 \mathrm{kHz}$, the middle ear ossicles would dominate the response for $\mathrm{BC}$ at this frequency region.

The importance of the middle ear ossicles for BC perception have been estimated by computations (Stenfelt, 2006) and direct measurements (Röösli et al., 2012). Both these studies indicate the middle ear ossicles to be around $10 \mathrm{~dB}$ below other contributors for BC sound in the human when the stimulation was at the mastoid. Those results are in line with the predictions of the model in the current study (Fig. 5).

The two contributors that showed least importance according to the simulations was the sound pressure in the ear canal and the contribution from the intracranial sound pressure. The 
contribution from the intracranial sound pressure shows a steep decline at frequencies above $0.5 \mathrm{kHz}$ but is close to the others at lower frequencies. The data were only available down to $0.3 \mathrm{kHz}$ and it is not known if it is more important at the lower frequencies. The steep decline at higher frequencies depend on the increased impedance through the aqueducts between the cranial space and the inner ear (the VA and CA). During the simulations, the importance of the CA was investigated by adding the intracranial sound pressure ( $P_{I C}$ in Fig. 2$)$ in-phase or 180 degrees out-of-phase with the sound pressure at the VA. There was no visible difference in the results and the importance of the CA for the intracranial sound pressure is negligible according to the current simulations.

The intracranial sound pressure has been argued to be important for BC hearing in the human, at least when the stimulation is at the soft tissues that connect to the cranial cavity as the eye or direct application of the transducer to the dura (Ito et al., 2011; Sohmer et al., 2000). However, in a study by (Roosli et al., 2015) where the intracranial sound pressure and cochlear promontory vibration were measured in cadaver heads, there were little difference in the ratio between promontory motion and intracranial pressure for different stimulation positions as the mastoid and the eye. Consequently, that study indicates that the $\mathrm{BC}$ responses at eye stimulation is equally likely to be due to bony vibrations as the stimulation directly on the mastoid bone.

In the study on ear canal sound pressure during BC stimulation by Stenfelt et al. (2003b), it was estimated that the importance of the ear canal sound pressure for $\mathrm{BC}$ hearing in the human was some 10 to $20 \mathrm{~dB}$ below the contribution from the middle ear. That is also predicted by the model simulations up to frequencies of $4 \mathrm{kHz}$, at the highest frequencies the model predicts less contribution from the ear canal sound pressure. According to Huizing (1960), the ear canal sound pressure increase at low frequencies is 10 to $15 \mathrm{~dB}$ greater than the perceived occlusion effect (e.g. as measured by hearing thresholds). That would indicate 
that at low frequencies, the ear canal sound pressure contribution should be 10 to $15 \mathrm{~dB}$ below the total $\mathrm{BC}$ contribution. The model predicts this difference to be 10 to $20 \mathrm{~dB}$ except for the very low frequencies, below $200 \mathrm{~Hz}$. However, in Stenfelt et al. (2007) the difference between ear canal sound pressure and perceived occlusion effect was less than $10 \mathrm{~dB}$, which was less than the model predicts. No data with occluded ear canals were used in the simulation but may serve as a validation tool for further model development where occluded ear canal sound pressure and perceived occlusion effect are used.

The data used here are obtained with stimulation in the area of the mastoid or slightly further back, at the stimulation position for a bone conduction hearing aid. This means that the exact stimulation position for the different datasets can differ by up to $20 \mathrm{~mm}$. With the transducer applied to the mastoid, small differences in stimulation positions have been reported to result in large sensitivity differences in the human for single frequencies (von Békésy, 1960).

However, for the frequency range used here, the sensitivity difference between a position at the mastoid and one around $20 \mathrm{~mm}$ further back was on average $2 \mathrm{~dB}$ (Stenfelt, 2012). It is therefore felt that the small differences in exact stimulation positions between datasets used for the simulation has only given a minor uncertainty to the results presented.

One issue that may affect the predictions made by the model is that the datasets used were obtained using different methodologies. Most BC vibration data were obtained in cadaver heads (Eeg-Olofsson et al., 2008; Stenfelt et al., 2005a) but live human data were also used (Eeg-Olofsson et al., 2013). Moreover, hearing threshold data were from measurements in live humans. Other data were obtained in temporal bone specimens (Stenfelt et al., 2002) where the vibration pattern may be different from intact heads. Most data are vibrations that were measured with laser Doppler vibrometers or accelerometers in a specific direction but some measurements were of sound pressure (Aibara et al., 2001; Roosli et al., 2015; Stenfelt et al., 2003b) that has no associated directional sensitivity. All these differences may affect 
the estimated importance of the contributors. The results should be interpreted with this in mind.

\subsection{Clinical relevance}

The results presented here are simulations of a simplified model, and, as stated above, should be interpreted with care. However, some indications from the simulations can be drawn. It was found that there were three contributors within $10 \mathrm{~dB}$ for almost the entire frequency range. This implies that as long as one of these is unaltered, there are very small changes in the BC sensitivity. For example, the depression in BC sensitivity at around $2 \mathrm{kHz}$ when the stapes is immobilized as in otosclerosis has been attributed to the lack of middle ear inertia. However, the simulations here do not indicate that removal of the outer ear and middle ear component would result in a frequency specific reduction of $20 \mathrm{~dB}$ in $\mathrm{BC}$ sensitivity. It is more likely, as indicated in Stenfelt (2015), that the changed BC sensitivity is a result of removal of the middle ear component together with alteration of the impedance at the OW leading to less efficient contribution from the inner ear components. Similar results are shown in finite element models of the inner ear with BC stimulation (Kim et al., 2011; Kim et al., 2014a)

Another indications from the simulations is the importance of the intracranial pressure for BC excitation. It has been argued that excitation directly of the soft tissues lead to direct excitation of the inner ear via patent pathways (Adelman et al., 2013). However, the simulations here indicate that, unless the BC hearing thresholds are very depressed at the high frequencies, bone vibration plays an important role for BC hearing at these stimulation sites as well. 


\section{Conclusions}

An inner ear model based on impedances of the compartments and inlets and outlets of the inner ear together with data on BC sound transmission to the cochlear promontory, BC generated ear canal sound pressure, sound transmission through the middle ear, and BC generated intracranial sound pressure, were used to predict the importance of five contributors for BC perception in the human. The model predicts the three components middle ear inertia, inner ear fluid inertia, and inner ear compression to be the most important. Of these three, the inner ear fluid inertia seem to be most important. The two least important contributors were sound pressure in the ear canal and BC generated intracranial sound pressure. The ear canal sound pressure was 20 to $35 \mathrm{~dB}$ below the total contribution at the low and high frequencies, while it was around $15 \mathrm{~dB}$ below at mid-frequencies. Intracranial sound pressure seem to be close to the three most important contributors at the very low frequencies, but decline with frequency and was more than $50 \mathrm{~dB}$ below the total contribution at $10 \mathrm{kHz}$. The model for BC excitation was compared with AC excitation at hearing thresholds. That comparison showed, except for the lowest frequencies, the model to be acceptable for frequencies up to $4 \mathrm{kHz}$. Above $4 \mathrm{kHz}$ the difference was significant between $\mathrm{AC}$ and $\mathrm{BC}$ driven $\mathrm{BM}$ motion at hearing thresholds. 


\section{Acknowledgement}

This work was supported by the European Union under Grant No. 600933 for the SIFEM project.

\section{References}

Adelman, C., Sohmer, H. 2013. Thresholds to soft tissue conduction stimulation compared to bone conduction stimulation. Audiology and Neurotology 18, 31-35.

Aibara, R., Welsh, J., Puria, S., Goode, R. 2001. Human middle ear sound transfer function and cochlear input impedance. Hearing Research 152, 100-109.

Bárány, E. 1938. A contribution to the physiology of bone conduction. Acta Oto-laryngologica Supplementum 26, 1-223.

Bohnke, F., Arnold, W. 2006. Bone conduction in a three-dimensional model of the cochlea. ORL; journal for oto-rhino-laryngology and its related specialties 68, 393-396.

Brandt, A. 1989. On sound transmission characteristics of the human skull in vivo. Technical report 61L. Chalmers University of Technology, Göteborg.

Braun, K., Böhnke, F., Stark, T. 2012. Three-dimensional representation of the human cochlea using micro-computed tomography data: presenting an anatomical model for further numerical calculations. Acta Otolaryngologica 132, 603-613.

Brummund, M., Sgard, F., Petit, Y., Laville, F. 2014. Three-dimensional finite element modeling of the human external ear: simulation study of the bone conduction occlusion effect. J. Acoust. Soc. Am. 135, 1433-1444.

Carhart, R. 1971. Effects of stapes fixation on bone-conductio response. In: Ventry, I., Chaiklin, J., Dixon, R., (Eds.), Hearing Measurement: A Book of Readings. Appleton, Century, Crofts, New York. pp. 116-129.

Clemis, J., Valvassori, G. 1968. Recent radiographic and clinical observation on vestibular aqueduct (a preliminary report). Otolaryngologic Clinics of North America 1, 339-46. 
Eeg-Olofsson, M., Stenfelt, S., Tjellström, A., Granström, G. 2008. Transmission of bone-conducted sound in the human skull measured by cochlear vibrations. International Journal of Audiology 47, 761-769.

Eeg-Olofsson, M., Stenfelt, S., Taghavi, H., Reinfeldt, S., Hakansson, B., Tengstrand, T., Finizia, C. 2013. Transmission of bone conducted sound - Correlation between hearing perception and cochlear vibration. Hearing Research 306, 11-20.

Franke, E. 1956. Response of the human skull to mechanical vibrations. J. Acoust. Soc. Am. 28, 12771284.

Gopen, Q., Rosowski, J., Merchant, S. 1997. Anatomy of the normal human cochlear aqueduct with functional implications. Hearing Research 107, 9-22.

Hato, N., Stenfelt, S., Goode, R.L. 2003. Three-dimensional stapes footplate motion in human temporal bones. Audiology and Neuro-Otology 8, 140-152.

Herzog, H. 1926. Das knochenleitungsproblem. Theoretische erwagungen. Zeitschreift fur Hals, Nasen und Ohrenheilk 15, 300-306.

Homma, K., Du, Y., Shimizu, Y., Puria, S. 2009. Ossicular resonance modes of the human middle ear for bone and air conduction. J. Acoust. Soc. Am. 125, 968-979.

Huber, A., Linder, T., Ferrazzini, M., Schmid, S., Dillier, N., Stoeckli, S., Fisch, U. 2001. Intraoperative assessment of stapes movement. Annals of Otology Rhinology and Laryngology 110, 31-35.

Huizing, E. 1960. Bone conduction - The influence of the middle ear. Acta Oto-Laryngolica Supplementum 155, 1-99.

ISO:389-3. 1994. International Organization for Standardization Reference zero for the calibration of audiometric equipment. Part 3: Reference equivalent threshold force levels for pure tones and bone vibrators, Geneva.

Ito, T., Röösli, C., Kim, C., Sim, J., Huber, A., Probst, R. 2011. Bone conduction thresholds and skull vibration measured on the teeth during stimulation at different sites on the human head. Audiology Neurootology 16, 12-22. 
Khanna, S.M., Tonndorf, J., Queller, J. 1976. Mechanical parameters of hearing by bone conduction. J. Acoust. Soc. Am. 60, 139-154.

Killion, M.C. 1978. Revised estimate of minimum audible pressure: Where is the "missing 6 dB"? J. Acoust. Soc. Am. 63, 1501-1508.

Kim, N., Homma, K., Puria, S. 2011. Inertial bone conduction: Symmetric and anti-symmetric components. Journal of the Association for Research in Otolaryngology 12, 261-279.

Kim, N., Steele, C., Puria, S. 2014a. The importance of the hook region of the cochlea for boneconduction hearing. Biophysical Journal 107, 233-241.

Kim, N., Chang, Y., Stenfelt, S. 2014b. A 3D finite element model of a human dry skull for a boneconducted hearing. BioMed Research International 519429, 1-9.

Krainz, W. 1926. Das knochenleitungsproblem. Experimentelle Ergebnisse. Zeitschreift fur Hals, Nasen und Ohrenheilk 15, 306-313.

McKnight, C., Doman, D., Brown, M., Bance, M., Adamson, R. 2013. Direct measurement of the wavelength of sound waves in the human skull. J. Acoust. Soc. Am. 133, 136-145.

Merchant, S., Ravicz, M., Rosowski, J. 1996. Acoustic input impedance of the stapes and cochlea in human temporal bones. Hearing Research 97, 30-45.

Purcell, D., Kunov, H., Madsen, P., Cleghorn, W. 1998. Distortion product otoacoustic emissions stimulated through bone conduction. Ear and Hearing 19, 362-370.

Purcell, D., Johnson, J., Fishbein, N., Lalwani, A. 2003. Establishment of normative cochlear and vestibular measurements to aid in the disgnosis of inner ear malformations. Otolaryngology - Head and Neck Suregery 128, 78-87.

Puria, S. 2003. Measurements of human middle ear forward and reverse acoustics: Implications for otoacoustic emissions. J. Acoust. Soc. Am. 113, 2773-2789.

Puria, S., Peake, W., Rosowski, J. 1997. Sound-pressure measurements in the cochlear vestibule of human-cadaver ears. Journal of the Acoustical Society of America 101, 2754-2770. 
Reinfeldt, S., Stenfelt, S., Håkansson, B. 2013. Estimation of bone conduction skull transmission by hearing thresholds and ear-canal sound pressure. Hearing Research 299, 19-28.

Roosli, C., Dobrev, I., Sim, J., Gerig, R., Pfiffner, F., Stenfelt, S., Huber, A. 2015. Intracranial pressure and promontory vibration with soft tissue stimulation in cadaveric human whole heads. Otology and Neurotology, In press.

Ruggero, M., Temchin, A. 2003. Middle-ear transmission in humans: wide-band, not frequencytuned? Acoustics Research Letters Online 4, 53-58.

Röösli, C., Chhan, D., Halpin, C., Rosowski, J. 2012. Comparison of umbo velocity in air- and boneconduction. Hearing Research 290, 83-90.

Schick, F. 1991. An electrical model for the simulation of bone and air conducted hearing. Acustica 74, 134-142.

Schroeter, J., Poesselt, C. 1986. The use of acoustical test fixtures for the measurement of hearing protector attenuation. Part II: Modeling the external ear, simulating bone conduction, and comparing test fixture and real-ear data. J. Acoust. Soc. Am. 80, 505-527.

Seibel, V., Lavinsky, L., Irion, K. 2006. CT-Scan sheep and human inner ear morphometric comparison. Brazilian Journal of Otorhinolaryngology 72, 370-376.

Sohmer, H., Freeman, S., Geal-Dor, M., Adelman, C., Savion, I. 2000. Bone conduction experiments in humans - a fluid pathway from bone to ear. Hearing Research 146, 81-88.

Stenfelt, S. 2006. Middle ear ossicles motion at hearing thresholds with air conduction and bone conduction stimulation. J. Acoust. Soc. Am. 119, 2848-2858.

Stenfelt, S. 2007. Simultaneous cancellation of air and bone conduction tones at two frequencies: extension of the famous experiment by von Békésy. . Hearing Research 225, 105-116.

Stenfelt, S. 2011. Acoustic and physiologic aspects of bone conduction hearing. Advances in OtoRhino-Laryngology 71, 10-21. 
Stenfelt, S. 2012. Transcranial attenuation of bone conducted sound when stimulation is at the mastoid and at the bone conduction hearing aid position. Otology and Neurotology 33, 105114.

Stenfelt, S. 2015. Inner ear contribution to bone conduction hearing in the human. Hearing Research, in press.

Stenfelt, S., Goode, R.L. 2005a. Transmission properties of bone conducted sound: Measurements in cadaver heads. J. Acoust. Soc. Am. 118, 2373-2391.

Stenfelt, S., Goode, R. 2005b. Bone conducted sound: Physiological and clinical aspects. Otology and Neurotology 26, 1245-1261.

Stenfelt, S., Reinfeldt, S. 2007. A model of the occlusion effect with bone-conducted stimulation. International Journal of Audiology 46, 595-608.

Stenfelt, S., Håkansson, B., Tjellström, A. 2000. Vibration characteristics of bone conducted sound in vitro. J. Acoust. Soc. Am. 107, 422-431.

Stenfelt, S., Hato, N., Goode, R. 2002. Factors contributing to bone conduction: The middle ear. J. Acoust. Soc. Am. 111, 947-959.

Stenfelt, S., Hato, N., Goode, R.L. 2004. Fluid volume displacement at the oval and round windows with air and bone conduction stimulation. J. Acoust. Soc. Am. 115, 797-812.

Stenfelt, S., Puria, S., Hato, N., Goode, R.L. 2003a. Basilar membrane and osseous spiral lamina motion in human cadavers with air and bone conduction stimuli. Hearing Research 181, 131143.

Stenfelt, S., Wild, T., Hato, N., Goode, R.L. 2003b. Factors contributing to bone conduction: The outer ear. J. Acoust. Soc. Am. 113, 902-912.

Taschke, H., Hudde, H. 2006. A finite element model of the human head for auditory bone conduction simulation. ORL; journal for oto-rhino-laryngology and its related specialties 68 , 319-323. 
Tonndorf, J. 1966. Bone Conduction. Studies in Experimental Animals. Acta Oto-Laryngologica Suppl 213, 1-132.

Tonndorf, J., Jahn, A.F. 1981. Velocity of propagation of bone-conducted sound in a human head. J. Acoust. Soc. Am. 70, 1294-1297.

Watanabe, T., Bertoli, S., Probst, R. 2008. Transmission pathways of vibratory stimulation as measured by subjective thresholds and distortion-product otoacoustic emissions. Ear and Hearing 29, 667-673.

Williams, M., Howell, P. 1990. An Electrical Network Model of Inertially Induced Bone-Conducted Sound. Scand. Audiol. 19, 161-170.

von Békésy, G. 1932. Zur Theorie des Hörens bei der Schallaufnahme durch Knochenleitung. Ann. Physik 13, 111-136.

von Békésy, G. 1960. Experiments in hearing McGraw - Hill, New York. 


\section{Tables}

Table 1. Impedances and the parameter values for the inner ear model.

\begin{tabular}{|c|c|}
\hline$Z_{V A}=j \omega \frac{M_{V}}{2}+Z_{V A_{-} D U C T}$ & \\
\hline$Z_{V}=j \omega \frac{M_{V}}{2}$ & \\
\hline$Z_{S V}=j \omega \frac{M_{S V}}{2}$ & \\
\hline$Z_{C}=j \omega \frac{M_{S V}}{2}+Z_{B M}+j \omega \frac{M_{S T}}{2}$ & \\
\hline$Z_{R W}=j \omega \frac{M_{S T}}{2}+\frac{K_{R W}}{j \omega}$ & \\
\hline$M_{S T}=9.17 \cdot 10^{5}\left[\mathrm{~kg} / \mathrm{m}^{4}\right]$ & (Stenfelt, 2015) \\
\hline$M_{S V}=4.89 \cdot 10^{5}\left[\mathrm{~kg} / \mathrm{m}^{4}\right]$ & (Stenfelt, 2015) \\
\hline$M_{V}=4.86 \cdot 10^{5}\left[\mathrm{~kg} / \mathrm{m}^{4}\right]$ & (Stenfelt, 2015) \\
\hline$Z_{M E}=j \omega \cdot 4.4 \cdot 10^{5}+10^{10}-j \cdot \frac{8.1 \cdot 10^{13}}{\omega}\left[\mathrm{kg} / \mathrm{m}^{4} \mathrm{~s}\right]$ & (Puria, 2003) \\
\hline$Z_{B M}=j \omega \cdot 3 \cdot 10^{5}+10^{10}\left[\mathrm{~kg} / \mathrm{m}^{4} \mathrm{~s}\right]$ & (Puria, 2003) \\
\hline$K_{R W}=7 \cdot 10^{12}\left[\mathrm{~kg} / \mathrm{m}^{4} \mathrm{~s}^{2}\right]$ & (Merchant et al., 1996) \\
\hline$Z_{V A_{-} D U C T}=j \omega \cdot 9.8 \cdot 10^{7}+2.1 \cdot 10^{10}-j \cdot \frac{4.3 \cdot 10^{12}}{\omega}\left[\mathrm{kg} / \mathrm{m}^{4} \mathrm{~s}\right]$ & (Stenfelt, 2015) \\
\hline$Z_{C A}=j \omega \cdot 7.5 \cdot 10^{8}+8.0 \cdot 10^{11}-j \cdot \frac{4.3 \cdot 10^{12}}{\omega}\left[\mathrm{kg} / \mathrm{m}^{4} \mathrm{~s}\right]$ & $\left(\right.$ Stenfelt, 2015) ${ }^{1}$ \\
\hline
\end{tabular}

${ }^{1}$ There was a typo in $Z_{c A}$ in Stenfelt (2015). The current $Z_{C A}$ is computed according to the dimensions given in Stenfelt (2015). 


\section{Figure legends}

Figure 1: A layout of the ear. The ear canal, middle ear, and inner ear are shown and three pathways are indicated. The $\mathrm{BC}$ stimulation is at the mastoid bone and pathway 1 (blue arrow) indicates the sound transmission for the ear canal sound pressure with sound generated to in the ear canal (1A) and subsequent transmission to the inner ear via the middle ear (1B). Pathway 2 (purple arrow) indicates the bone vibration transmission to the bone surrounding the inner ear and pathway 3 (red arrow) indicates the transmission for skull interior with intracranial sound pressure $(3 \mathrm{~A})$ and the subsequent transmission to the inner ear via the vestibular aqueduct (3B).

Figure 2: The impedance model of the inner ear. The details of the model is given in the text.

Figure 3: The volume displacement at the BM according to the model in Fig. 2 for the five contributors when the stimulation is $1 \mathrm{~N}$ at the mastoid.

Figure 4: The displacement of the cochlear promontory from three different studies. The stimulation is $1 \mathrm{~N}$ at the mastoid. The solid line (Stenfelt et al., 2005a) and dashed line (Eeg-Olofsson et al., 2008) are from measurements in cadaver heads with the BC transducer anchored in mastoid bone and their mean is used in the current study. The dotted line (Eeg-Olofsson et al., 2013) represents measurements in live humans where the $B C$ transducer is applied on the skin covered mastoid.

Figure 5: The relative importance of the five contributors. The total contribution from all is represented as zero $\mathrm{dB}$, and the individual contribution is related to this total contribution.

Figure 6: The relation between $\mathrm{AC}$ driven and $\mathrm{BC}$ driven $\mathrm{BM}$ excitation at hearing thresholds. $\mathrm{A}$ level above $0 \mathrm{~dB}$ means that the $\mathrm{AC}$ transmission generates larger $\mathrm{BM}$ excitation than the $\mathrm{BC}$ transmission at hearing thresholds. Results are interpolated for frequencies between threshold data. The solid line is the results for the results of the model in Fig. 2. The dashed 
line is the result when the $B C$ wave velocity is increased to $1000 \mathrm{~m} / \mathrm{s}$, and the dotted line is when the cochlear promontory motion of live humans according to Eeg-Olofsson et al. (2013) is used. 


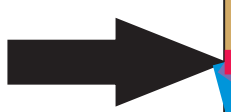

$3 A$

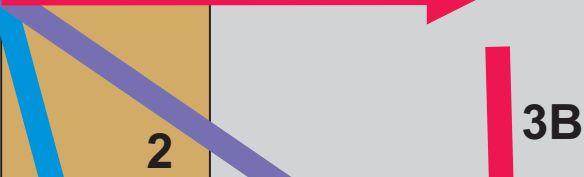

$1 \mathrm{~A}$

VA

Ear-canal

Skull-bone

CSF/Brain 
$3.4 \mathrm{~mm}$

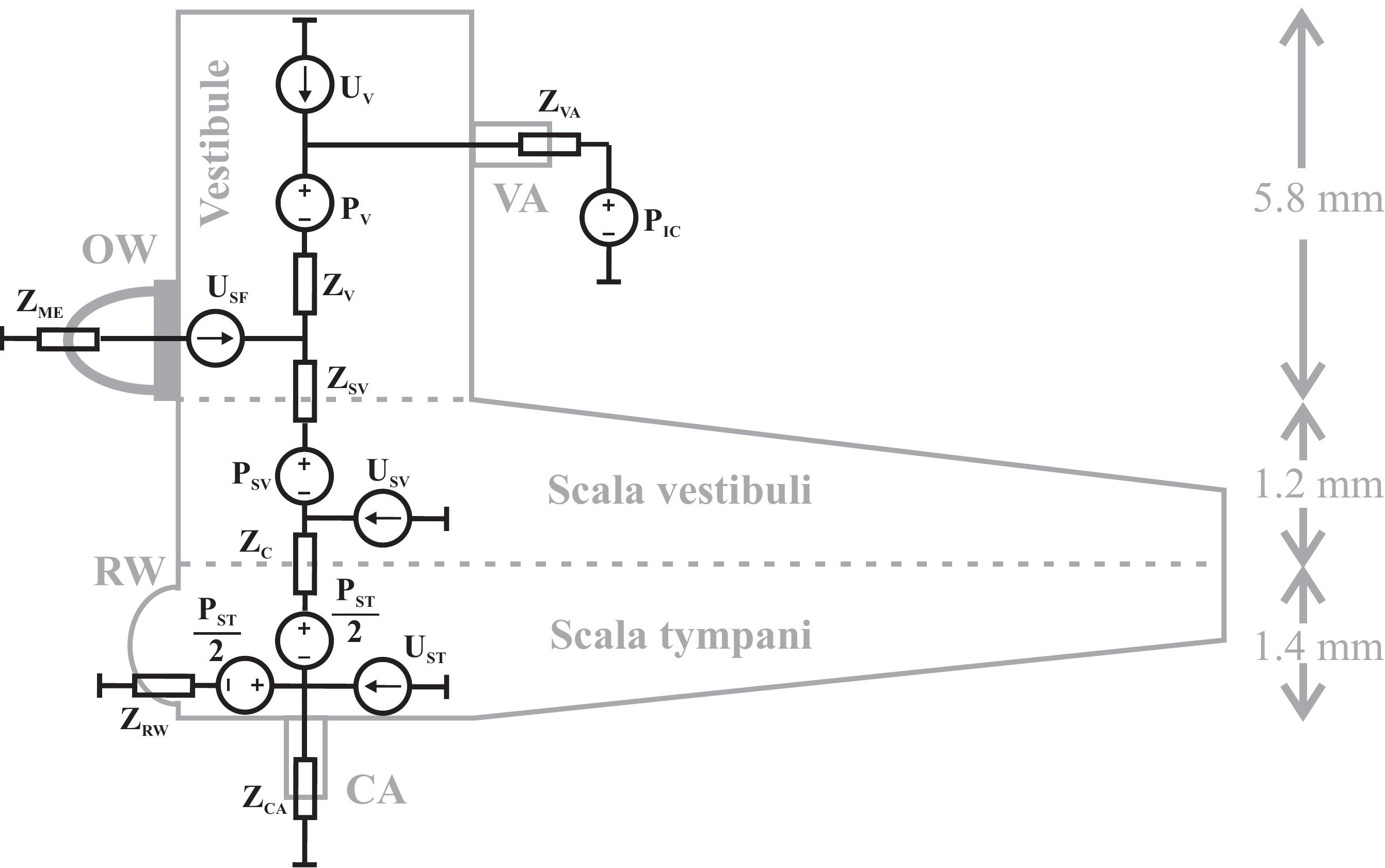




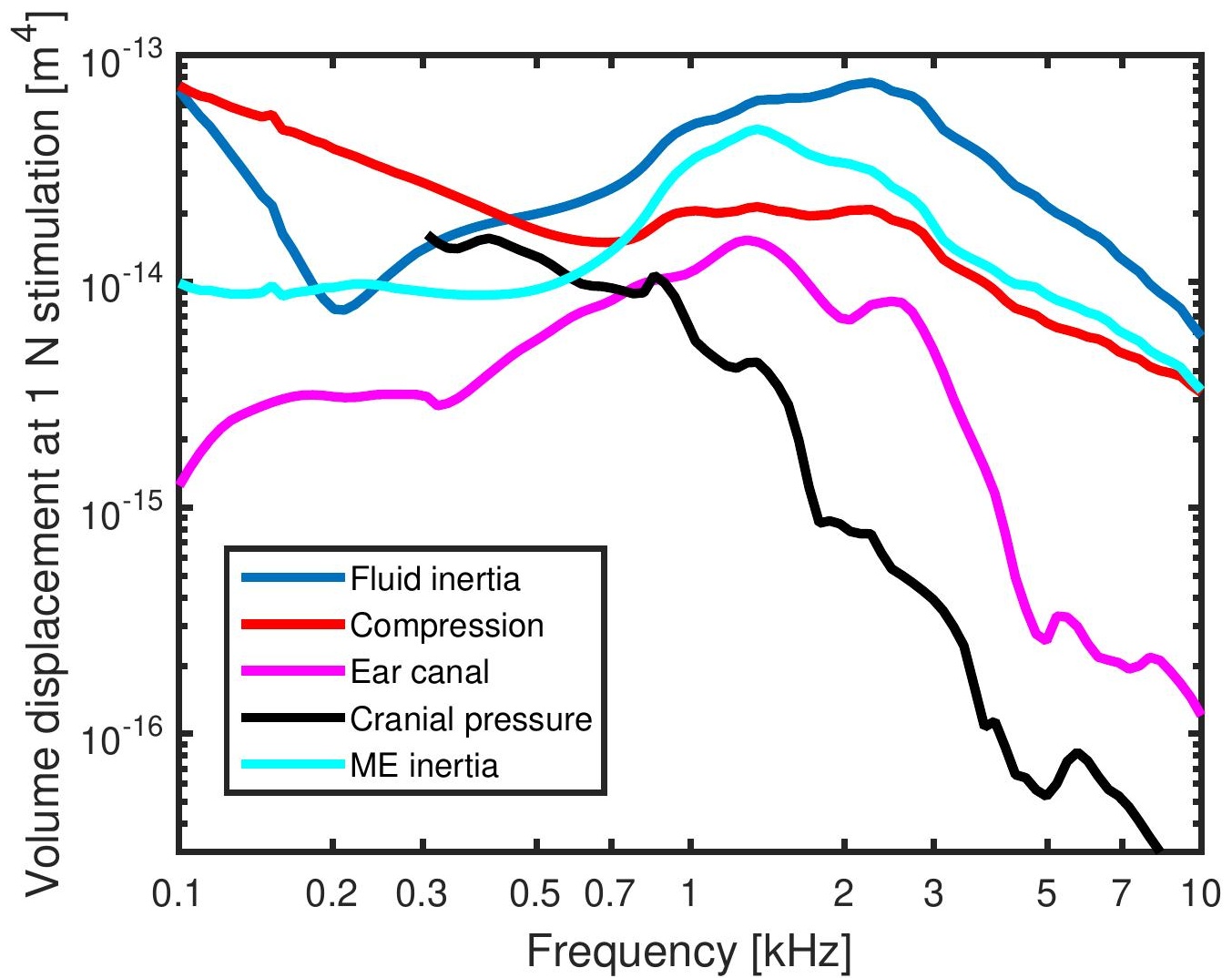




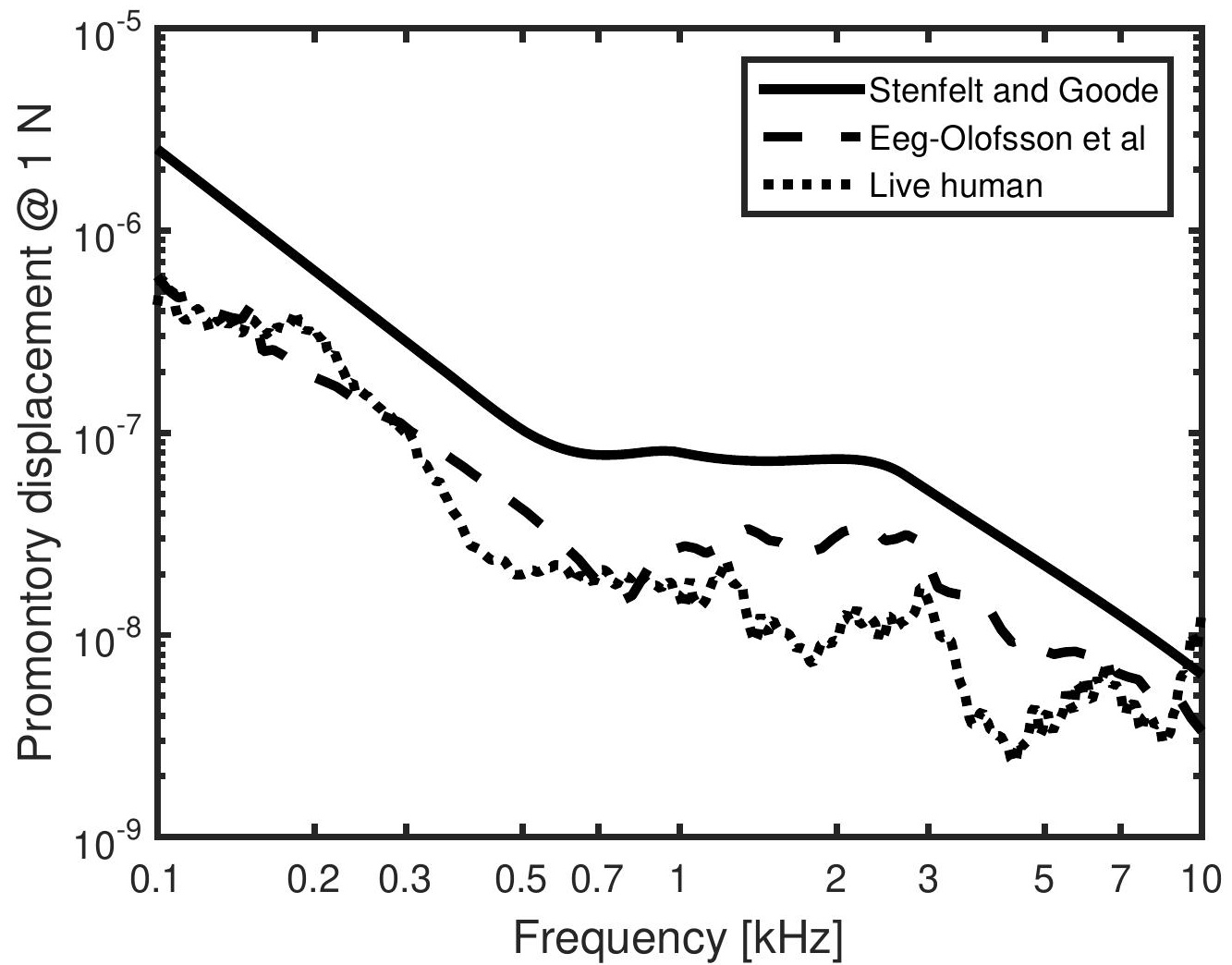




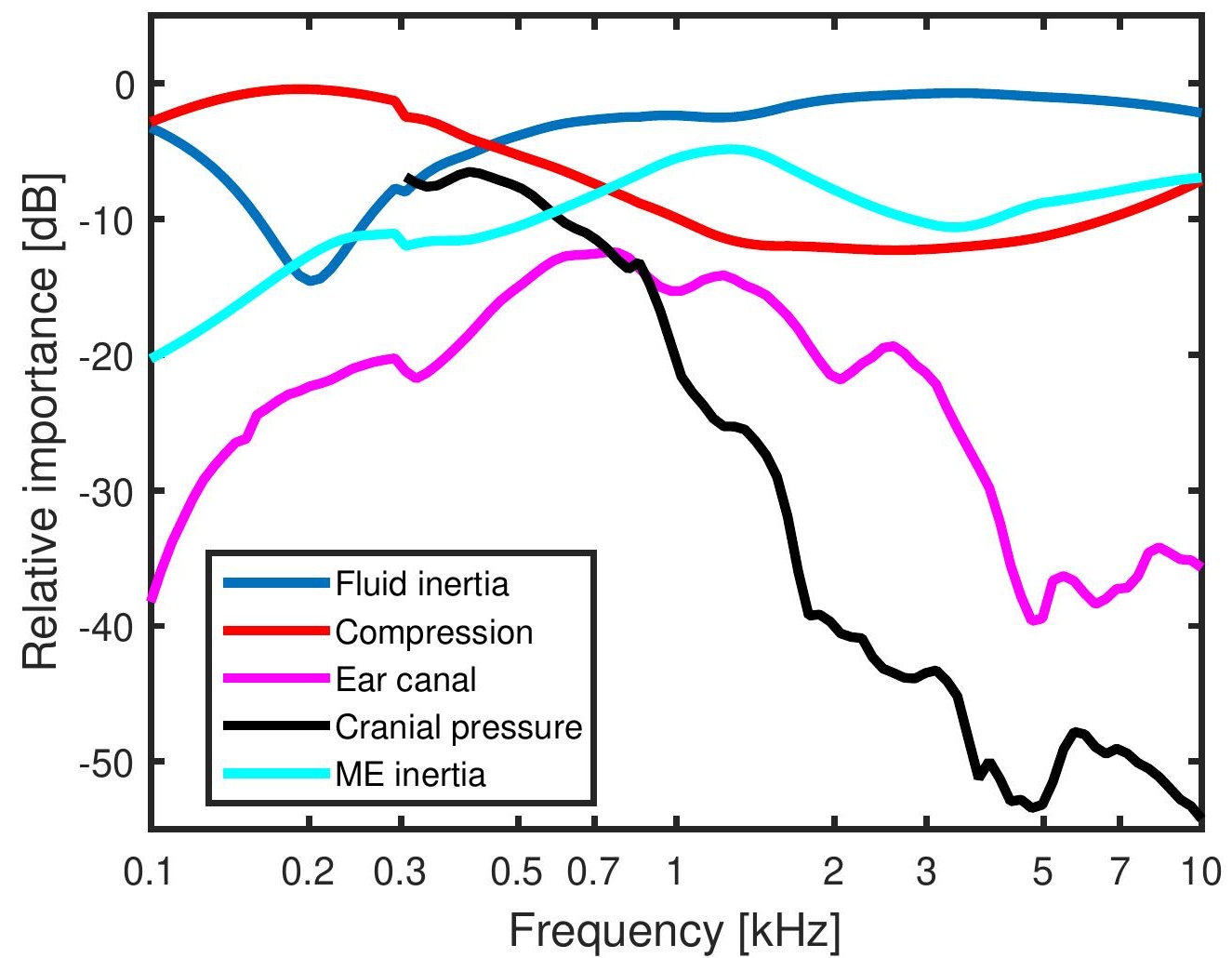




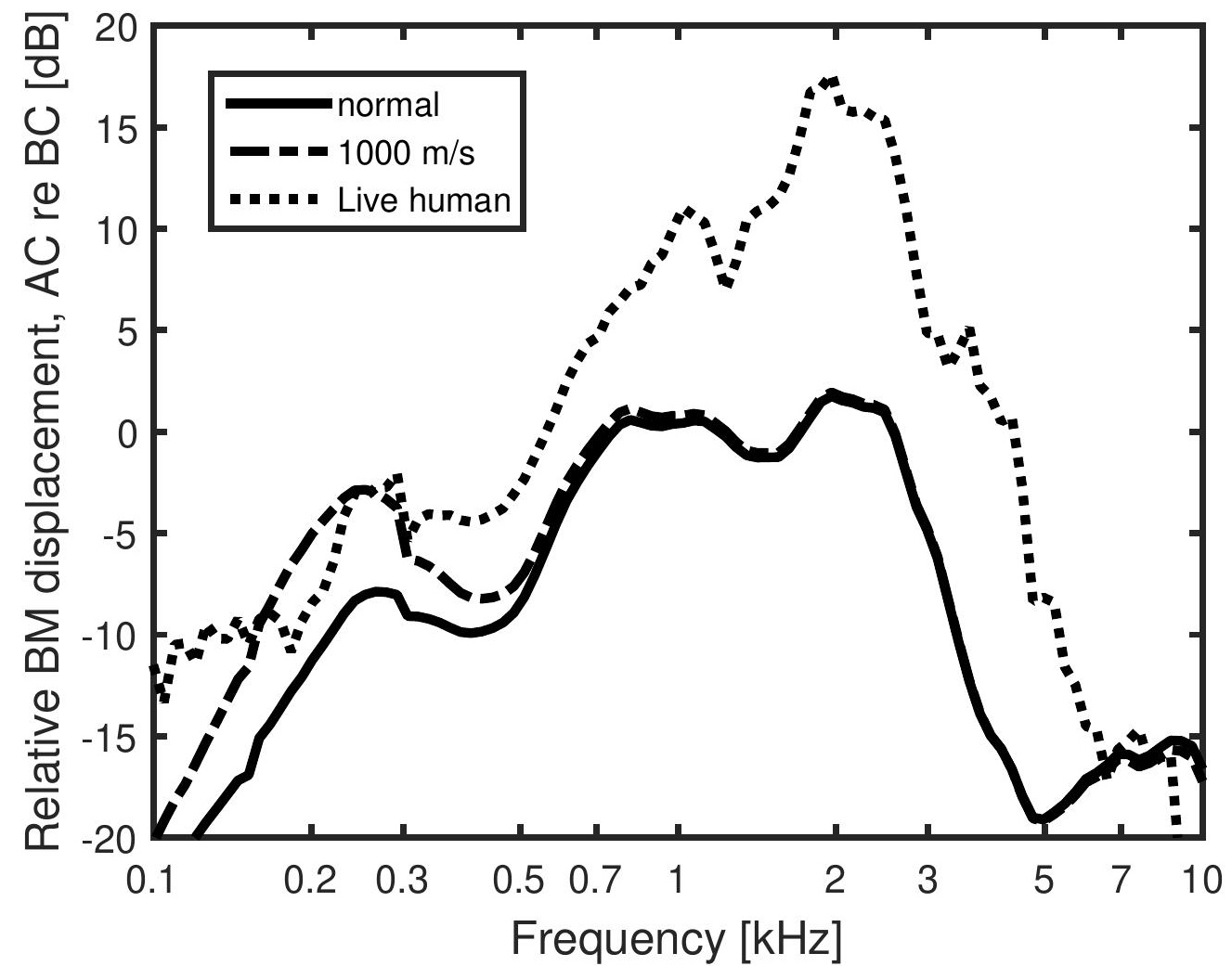

